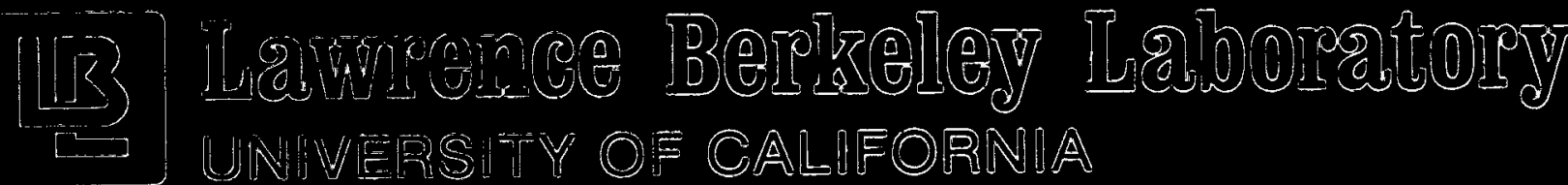

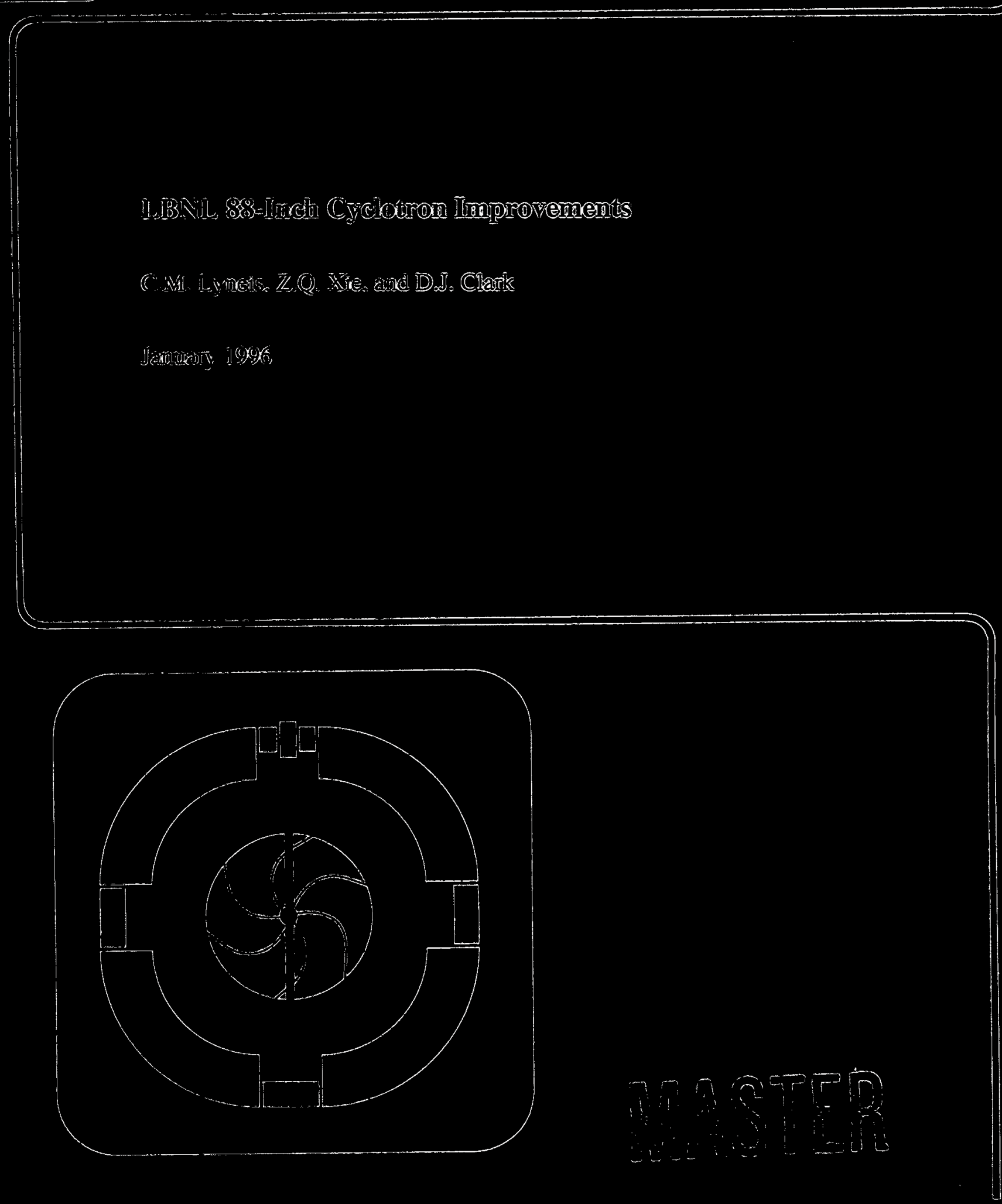

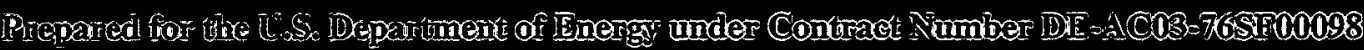

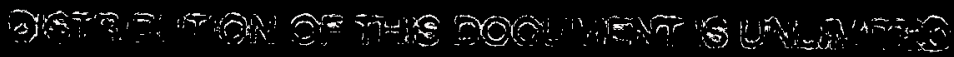


DISCLATYCBR

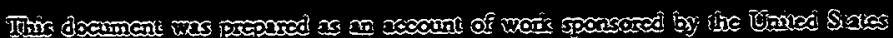

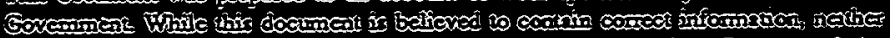

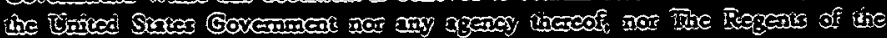

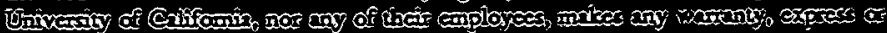

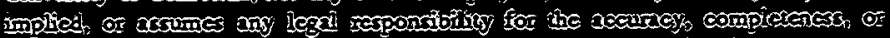

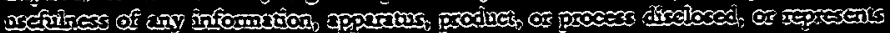

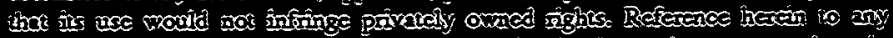

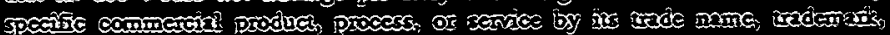

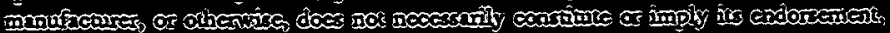

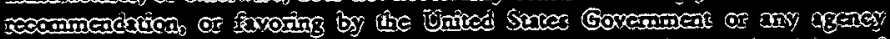

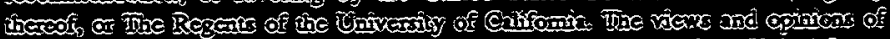

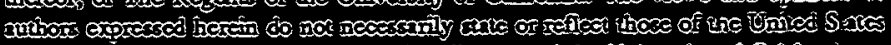

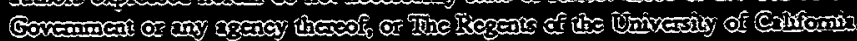

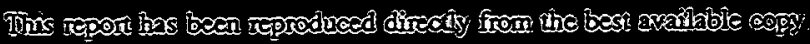

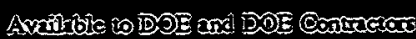

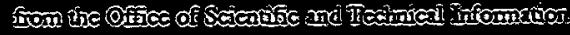

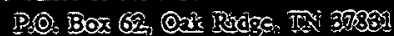

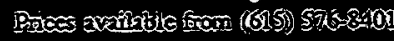

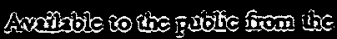

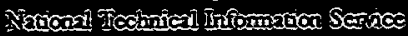

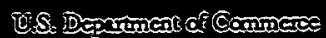

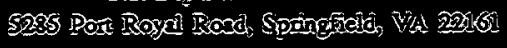

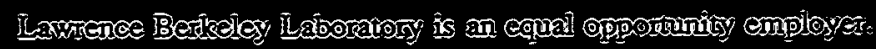


LBL - 38168

\title{
LBNL 88-INCH CYCLOTRON IMPROVEMENTS
}

\author{
C.M. Lyneis, Z.Q. Xie, D.J. Clark
}

Nuclear Science Division, Lawrence Berkeley National Laboratory

University of California, Berkeley, California 94720, USA

This work was supported by the Director, Office of Energy Research Division of Nuclear Physics of the Office of High Energy and Nuclear Physics of the U.S. Department of Energy under Contract DE-AC03-76SF00098

da 


\title{
LBNL 88-INCH CYCLOTRON IMPROVEMENTS
}

\author{
C.M. LYNEIS, Z.Q. XIE, DJ. CLARK
}

Lawrence Berkeley National Laboratory, I Cyclotron Rd, Berkeley, CA 94720, USA

\begin{abstract}
A number of recent improvements have been made in the Cyclotron, ECR sources and beam lines. An aluminum oxide wall coating in the AECR source produces more secondary electrons to increase high charge state ion production by $2-4$ compared to electron injection. Development of simultaneous multiple frequency ECR heating with 10 and $14 \mathrm{GHz}$ results in two ECR zones and increases the high charge state intensities by a factor of 2-5. The best transmission from the AECR source through the Cyclotron occurs for low energy first harmonic beams, where it varies with intensity from over $25 \%$ at low intensity $(10 \mu \mathrm{A}$ injected) to $12 \%$ at high intensity ( $180 \mu \mathrm{A}$ injected). A double drift buncher was built by adding a second harmonic drift tube after the existing one for Ist harmonic. This has produced up to $40 \%$ increase in extemal beam. New beam lines have been set up for nuclear structure experiments with Gammasphere and laser trapping of radioactive atoms. The new Gammasphere dispersion-compensated beam line transports up to $60 \%$ of the beam coming through a collimator at the cyclotron exit to a $3 \mathrm{~mm}$ diameter beam spot at the target $50 \mathrm{~m}$ downstream. Improvements now under way include a pulsing system for the axial injection line, a second cryopumping system for the cyclotron, phase width reduction using internal slits and the design of a 3rd generation ECR source.
\end{abstract}

\section{Introduction}

The 88-Inch Cyclotron is now operating with beams of higher energy, greater intensity and variety, with more hours of beam on target and supporting a larger number of users than any time since it began operation in 1962. At a time when many small accelerators are being closed down it may seem paradoxical that the cyclotron and its scientific program are doing so well. There are two major reasons for this. First, the development of the ECR ion source'by Geller et al $^{1}$ and its subsequent developmen $t^{2}$ have made it possible to produce a wide range of beams with intensities and energies well matched to the study of nuclear structure, nuclear reaction mechanisms and heavy element research. In addition the 88-Inch Cyclotron which began its operation as a light-ion cyclotron has maintained this capability, which provides research opportunities in new areas such a laser atom trapping for the study of weak interactions and fundamental symmetries. The second reason is the development of a new generation of high energy resolution detectors such as Gammasphere and Eurogam which has opened up a new area of research in the study of highly deformed nuclei. Much of the research with these new detectors involves the formation of rapidly rotating compound nuclei. Production of the compound nuclei is typically done by using projectiles with $9 \leq A<80$ with 4 to 6 $\mathrm{MeV} /$ nucleon. In many cases to produce the desired compound nucleus neutron rich beams such as ${ }^{36} \mathrm{~S}$ or ${ }^{48} \mathrm{Ca}$ are required. This research puts strong demands of the ion source capabilities both with respect to making beams from almost every element up to mass 80 and to be able to use separated isotopes in an economical way. For example, current cost of ${ }^{36} \mathrm{~S}$ and ${ }^{48} \mathrm{Ca}$ are about $\$ 47,000 / \mathrm{g}$ and $\$ 250,000 / \mathrm{g}$, respectively.

\section{Accelerator description}

The 88-Inch Cyclotron is nominally a k140 cyclotron with three sectors and three external ion sources. The main magnet can be operated to produce $k 160$ operation, but the transmission efficiency decreases rapidly above $k 140$ due to defocusing of the beam at extraction and in most cases with heavy ions it is better to use a higher charge state from the $E C R$ ion sources rather than operating $k$ values above 140 . The cyclotron RF frequency can be varied from $5.5 \mathrm{MHz}$ to 16.2 $\mathrm{MHz}$ and both first and 3rd harmonic beams are routinely accelerated. Minimum and maximum energies in first harmonic are 6.1 and $55 \mathrm{MeV} / n u c l e o n$ and in third harmonic 0.7 to $6 \mathrm{MeV} /$ nucleon. Beams have also been accelerated using 5th, 7th and 9th harmonic, although the transmission efficiency decreases rapidly with the high harmonic numbers due to poor RF capture in the center region.

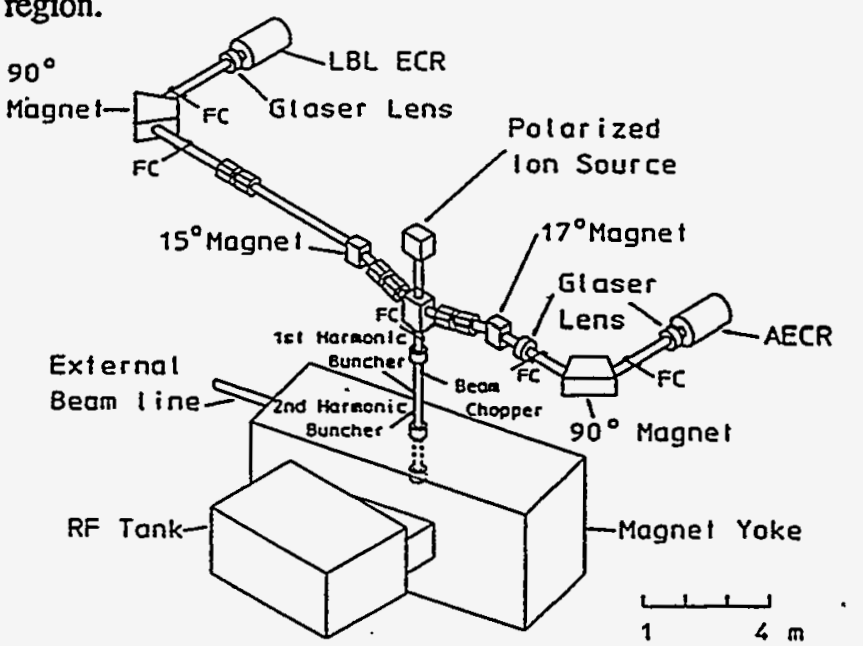

Fig. 1. Isometric view of the 88-Inch Cyclotron with external ion sources. 
Currently all beams being used by the research programs are produced by the two high charge state ECR ion sources located on the vault roof of the cyclotron as shown in Fig. 1. The LBL ECR ion source ${ }^{3}$ began operation with the cyclotron in 1985 and a second higher performance source the Advanced $\mathrm{ECR}^{4}$ was brought into operation in 1990. The horizontal beam lines for each source contain a double focusing 90 degree analyzing magnet with slits at the image and object for charge state selection. Ion beams from either source can be selected by reversing the 75 degree bending magnet at the top of the axial injection line and injected into the cyclotron. There are several advantages to having two ECR ion sources for the cyclotron. From the point of view of operations it allows "beam development" in terms of developing a specific ion beam from the ECR source without taking accelerator time. For difficult beams requiring ovens, isotopic feeds or maximum ion source performance the ion source can be brought on early and a rapid changeover to the next beam can be made. From the point of view of ion source development it is essential to have a second source especially when the cyclotron is operating 7 days a week with only one 8 hour shift for maintenance each week.

The axial beam line uses three solenoid lenses to transport the beam onto the gridded electrostatic inflector located on the axis of the cyclotron. Beam bunching is provided by a first harmonic buncher located $2.5 \mathrm{~m}$ above the cyclotron mid plane and a new second harmonic buncher located $0.4 \mathrm{~m}$ below the first harmonic buncher. The bunchers are both two gap devices with tungsten wire grids to provide the equipotential surfaces. The phase and amplitude of each buncher can be independently. adjusted to maximize transmission. Typical bunching factors are 3 for the first harmonic buncher and 4.5 for both bunchers. An axial line chopper was recently installed to provide chopping of the beam injected into the cyclotron. The present rise time is about $7 \mu$ s and modification of the drive circuits are underway to provide $1 \mu$ s rise time. This chopper is used for experiments with collection wheels and for experiments where the researchers need to control the integrated flux on target.

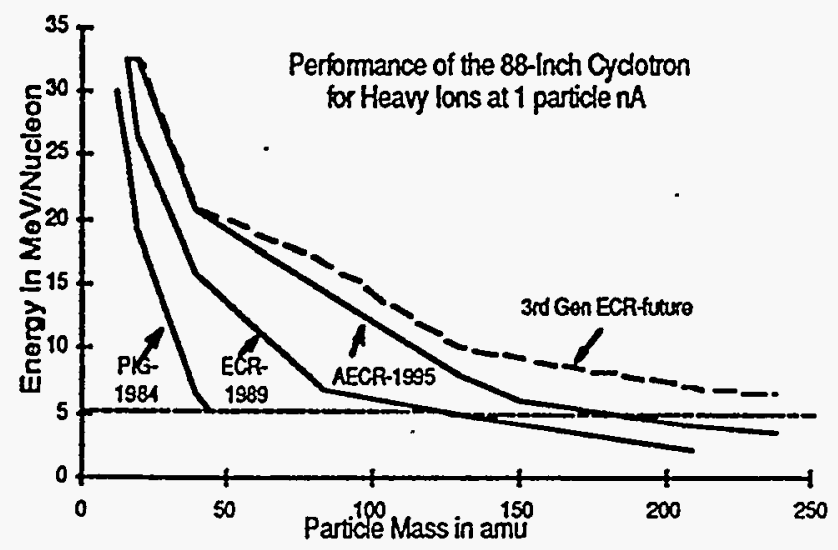

Fig. 2. This shows the energy mass curves for the cyclotron at an intensity of 1 particle $n A$.

\section{System performance}

The evolution of the heavy-ion performance of the 88-Inch Cyclotron is illustrated in Fig. 2. These curves are drawn for beams of at least 1 particle nA extracted current, which is a typical intensity required for nuclear structure experiments, and the dashed line at $5 \mathrm{MeV} /$ nucleon indicates the approximate cutoff in energies useful for nuclear science experiments. Higher intensity beams can be produced at energies less than those indicated by the curves. Prior to the operation with the first ECR ion source the heavy-ion operation was limited to elements with mass 40 or below. The LBL ECR began regular operation with the Cyclotron in January 1985 and it reached its full operating potential a few years later. With this source it was possible to accelerate masses up to xenon at useful intensities and energies. In 1990 the AECR began operation and with improvements in its performance described below it is now possible to reach masses up to 160. A conceptual design for a 3rd Generation ECR sources which would extend the energy mass characteristics of the cyclotron significantly is underway and the cyclotron's projected performance is indicated with a dashed line in Fig. 2.

Along with developing higher ion source performance with respect to producing higher charge states and higher intensities, the development of a wide range of beams has been an important aspect of providing new research opportunities at the cyclotron. Both the LBL ECR and the AECR have radial access ports provided by slots in the sextupole structure which allow ovens to be installed. The oven technology was first developed on the LBL ECR. ${ }^{6}$ Ovens are now in use with many ECR ion sources although they are typically mounted on axis and with maximum temperatures of $1500^{\circ} \mathrm{C}$. The high temperature oven developed for the ECR ion sources at Berkeley can operate up to $2100^{\circ} \mathrm{C}$ which can evaporate more elements. To date, the 88-Inch Cyclotron has produced beams of 41 elements, from hydrogen through zinc (the first 30 elements) as well as $\mathrm{Ge}, \mathrm{Kr}, \mathrm{Ag}, \mathrm{Sn}, \mathrm{Xe}, \mathrm{La}, \mathrm{Sm}, \mathrm{Tb}, \mathrm{Au}, \mathrm{Bi}$ and $\mathrm{U}$.

Efficient use of separated isotopic feed material has been developed with the ECR sources also. For example in FY94 more than 300 hours of research utilized ${ }^{48} \mathrm{Ca}$ beams. For these experiments, which required about 2 particle $n A$ on target, typical usage rates of $40 \%$ enriched ${ }^{48} \mathrm{Ca}$ were 20 to $30 \mu \mathrm{g} / \mathrm{hr}$. Several factors go into the overall efficiency including the source efficiency, the injection and extraction efficiency and beam transmission to the experimental target. In a typical experiment about 150 particle $n A$ of $\mathrm{Ca}^{10+}$ is produced by the ECR source (to minimize consumption), 15 particle $\mathrm{nA}$ is extracted from the cyclotron, and 5 particle $\mathrm{nA}$ is delivered to the Cave $4 \mathrm{c}$ where Gammasphere is located. This represents an order of magnitude improvement in transmission efficiency compared above that achieved in the first few years of operation the LBL ECR. The improved overall transmission is a result of improvements in the transport through the injection line, higher bunching efficiency with the second harmonic buncher, better cyclotron settings and improvements in the beam transport system to the cave. 
stripped Ar ions were produced. Figure 4 shows the best charge state distributions of ${ }^{238} \mathrm{U}$ obtained with singlefrequency and two-frequency heating from the AECR source. With about $15 \%$ more microwave power compared to the optimum single-frequency heating, two-frequency heating shifted the peak charge state from $33+$ to $36+$ and increased the intensity by a factor of 2 to 4 higher for charge states from $35+$ to $39+$.

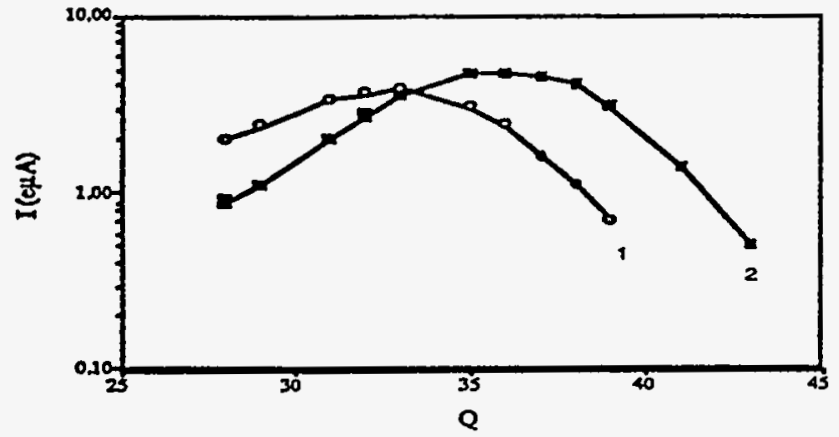

Fig. 4 Charge state distributions for ${ }^{238} \mathrm{U}$ produced with the AECR source are shown for single-frequency (14 GHz, curve 1) and two-frequency $(14+10 \mathrm{GHz}$, curve 2$)$ heating.

The improved performance of the AECR source with two-frequency heating makes it possible for the 88-Inch Cyciotron to accelerate the very heavy ions such as bismuth and uranium to an energy greater than $5 \mathrm{MeV} / \mathrm{n}$. Table $\mathrm{I}$ shows the highly charged ions obtained with the twofrequency heating and accelerated through the 88-Inch Cyclotron.

Table 1: Beams Produced by Two-frequency Heating and Accelerated by the 88-Inch Cyclotron

\begin{tabular}{lllc} 
Ion Extracted Cyclotron & \multicolumn{2}{c}{ Energy } \\
\multicolumn{2}{c}{ Current(enA) } & $(\mathrm{MeV})$ & $(\mathrm{MeV} / \mathrm{n})$ \\
\hline${ }^{86} \mathrm{KM}^{30+}$ & 0.22 & 1408.1 & 16.37 \\
${ }^{209} \mathrm{Bi}^{45+}$ & 0.5 & 1309.4 & 6.27 \\
${ }^{209} \mathrm{Bi}^{47+}$ & 0.33 & 1427.9 & 6.83 \\
${ }^{209} \mathrm{Bi}^{49+}$ & 0.021 & 1551.7 & 7.42 \\
${ }^{209} \mathrm{Bi}^{50+}$ & 0.015 & 1615.4 & 7.73 \\
${ }^{209} \mathrm{Bi}^{51+}$ & 0.004 & 1680.3 & 8.04 \\
${ }^{238} \mathrm{U}^{52+}$ & 0.017 & 1562.0 & 6.56 \\
${ }^{238} \mathrm{U}^{53+}$ & 0.008 & 1662.9 & 6.99 \\
\hline
\end{tabular}

An upgrade of the AECR is underway to modify its magnetic structure to improve the plasma confinement to further enhance the source performance. The existing solenoid magnets will be replaced by new ones with larger conductors and higher maximum current density. With these modifications and at the same total dc magnet power of 75 $\mathrm{kW}$ as the existing configuration, the peak field strengths at the injection and the extraction regions will increase about $70 \%$. The central field strength will remain the same which produces mirror ratios of 4.1 at injection and 3.0 at extraction. The wall thickness of the new aluminum plasma chamber wall be reduced and a stronger permanent sextupole magnet will be installed to increase the radial magnetic field at the wall from 0.64 to 1.0 Tesla. $^{13}$

A 3rd Generation ECR source is being designed to produce higher charge states and intensities so the 88-Inch Cyclotron can deliver useable heavy ion beams such as bismuth and uranium at energies above $5 \mathrm{MeV} /$ nucleon. ECR source development, especially in the last few years, indicates that further enhancement of the production of high charge state ions by ECR sources is possible with higher field strengths and higher magnetic mirror ratios, ${ }^{14}$ chamber surface with high secondary emission materials and heating the plasma with multiple frequency microwave power. ${ }^{12}$ The solenoid and sextupole magnets of this new source will be made from superconductor to produce the high magnetic field strengths. The design fields are more than $3 \mathrm{~T}$ in the axial direction and up to $2.5 \mathrm{~T}$ radial field at the plasma wall. This would provide mirror ratios of 15 at injection and 10 at extraction.

\section{Acknowledgments}

This work was supported by the Director, Office of Energy Research, Office of High Energy and Nuclear Physics, Nuclear Physics Division of the U.S. Department of Energy under Contract DE AC03-76SF00098.

\section{References}

1. Geller, R., Appl. Phys. Lett. 16, No 10, 401 (1970).

2. R. Geller, IEEE Trans. NS-26 No. 2, 2120 (1979).

3. C.M. Lyneis, Proceedings of the 6 th International Workshop on ECR Ion Source, Berkeley, CA, USA, 1986, p. 51.

4. Z.Q. Xie, C.M.Lyneis, R.S. Lam, and S.A. Lundgren, Rev. Sci. Instrum. 62, 775 (1991).

5. C.M. Lyneis and Z.Q. Xie, Proceedings of the 12th International Workshop on ECR Ion Sources, RIKEN, JAPAN, April 1995, p. 119.

6. D.J. Clark and C.M. Lyneis, J. Phys. (Paris) Colloq. 50, C1-759 (1989).

7. A.S. Schlachter, Proceedings of the 10th International Conference on Cyclotrons and Their Applications, E. Lansing, USA, April 1984, p. 563.

8. Z.Q. Xie and T.A. Antaya, Rev. Sci. Instrum., 61, 793 (1990).

9. T. Nakagawa et al, Jpn. J. Appl. Phys. 32, 1335 (1993).

10. Z.Q. Xie and C.M. Lyneis, Rev. Sci. Instrum. 65, (1994) 2947.

11. D. Hitz et al, Proceedings of the 12th International Workshop on ECR Ion Sources, RIKEN, JAPAN, April 1995, p. 126.

12. Z.Q. Xie and C.M Lyneis, Rev. Sci. Instrum. 66, 4218 (1995).

13. Z.Q. Xie and C.M. Lyneis, Proceedings of the 12th International Workshop on ECR Ion Sources, RIKEN, JAPAN, April 1995, p. 24.

14. T.A. Antaya and S. Gammino, Rev. Sci. Instrum. 65, 1723 (1994). 